\title{
PRIMARY CUTANEOUS NOCARDIOSIS- A DIAGNOSTIC DILEMMA
}

Nima Teresa Andrew ${ }^{1}$, Mercy Paul ${ }^{2}$

${ }_{1}^{1}$ Assistant Professor, Department of Dermatology, Jubilee Mission Medical College.

2Professor, Department of Dermatology, Jubilee Mission Medical College.

HOW TO CITE THIS ARTICLE: Andrew NT, Paul M. Primary cutaneous nocardiosis- a diagnostic dilemma. J. Evolution Med. Dent. Sci. 2017;6(95):7055-7057, DOI: 10.14260/jemds/2017/1530

\section{PRESENTATION OF CASE}

A 38-year-old male, working as a supermarket manager, developed multiple painful nodules on the face and arms associated with generalised weakness of 2 months duration. His medical history was otherwise uneventful. There was no previous history of any trauma or medication. He was investigated in a nearby hospital and was treated as cutaneous vasculitis with systemic corticosteroids. But there was no improvement in the symptoms.

Few weeks later, he developed similar new lesions on the chest and abdomen. Few lesions evolved to form an abscess, along with which he developed regional lymphadenopathy and fever. He consulted a different doctor and there he was diagnosed to have Cutaneous Tuberculosis and was started with Anti-Tuberculosis Therapy (ATT). Since there was no much improvement after 3 months of starting ATT, he was referred to our medical college.

On presentation, general examination revealed multiple tender, discrete lymph nodes of about $2 \times 1 \mathrm{~cm}$ size in the axilla, neck and groin.

\section{CLINICAL DIAGNOSIS}

On dermatological examination, he had multiple tender skin coloured to erythematous nodules of size varying from $2 \times 1$ $\mathrm{cm}$ to $5 \times 4 \mathrm{~cm}$ on the face, trunk, forearms, hand and a few lesions on the lower limbs [Figure 1,2]. Few nodules had dissolved to form abscess [Figure 3] and a few had ruptured to form crusted plaques [Figure 4].

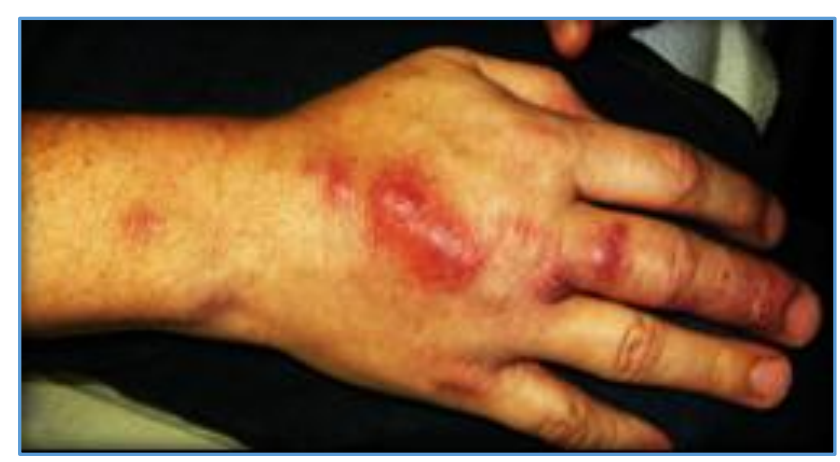

Figure 1. Erythematous Tender Nodules on the Dorsum of Hand

'Financial or Other Competing Interest': None.

Submission 11-10-2017, Peer Review 08-12-2017,

Acceptance 14-12-2017, Published 23-12-2017.

Corresponding Author:

Dr. Mercy Paul,

Professor,

Department of Dermatology,

Jubilee Mission Medical College.

E-mail: nimaandrew@gmail.com

DOI: $10.14260 / \mathrm{jemds} / 2017 / 1530$

(c) (i) (5) $\Theta$

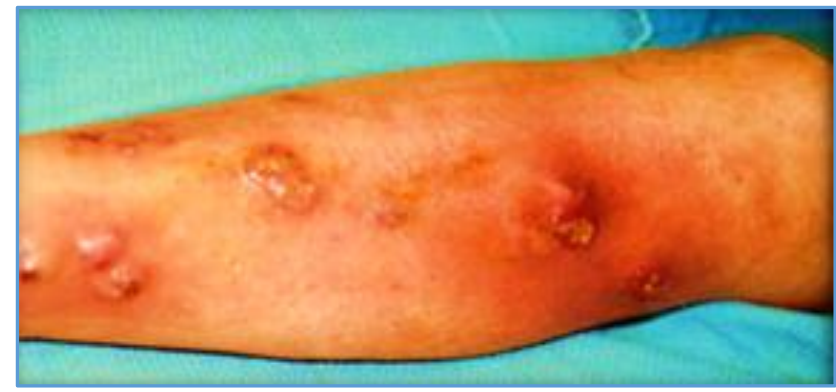

Figure 2. Multiple Erythematous Nodules on the Forearm

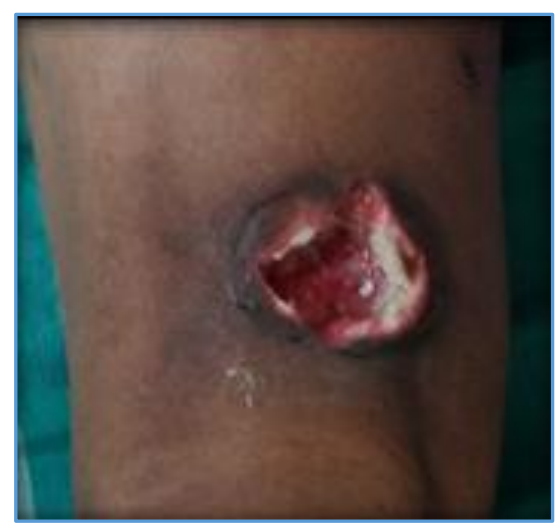

Figure 3. Ruptured Nodule with Abscess Formation on the Leg

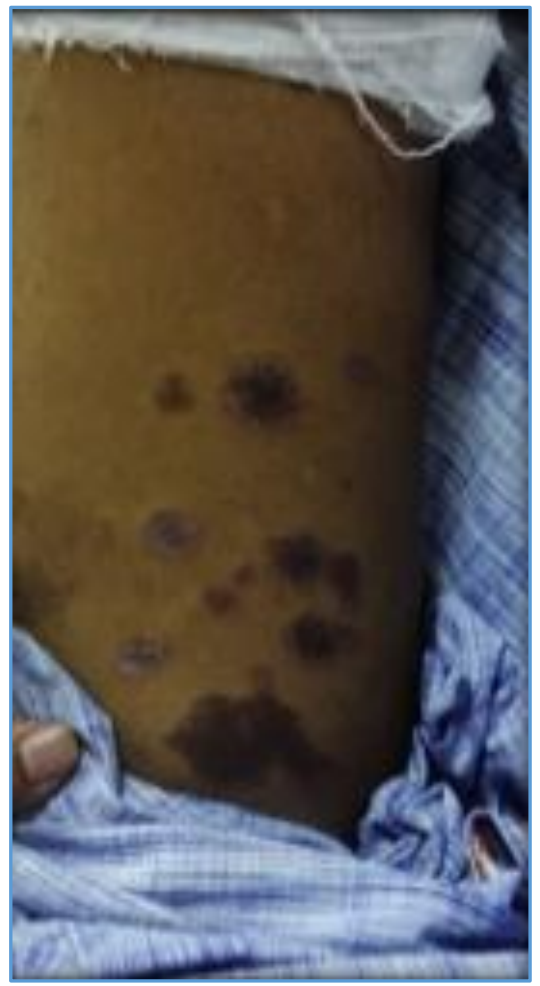

Figure 4. Multiple Crusted Plaques on the Trunk Systemic Examination was within Normal Limits 


\section{PATHOLOGICAL DISCUSSION}

Among the laboratory investigations, complete blood cell count showed leucocytosis along with neutrophilia $(14,300$ cells/ mm3 with $80 \%$ neutrophils) and a raised erythrocyte sedimentation rate (ESR) of $80 \mathrm{~mm} / \mathrm{hr}$. Liver and renal function tests and blood sugars were within normal limits. Screening for hepatitis B, C and HIV virus were negative.

Chest x-ray and electrocardiogram (ECG) were normal. An ultrasonogram of abdomen and computed tomography (CT scan) of brain and chest were taken and were found to be normal indicating that there was no systemic involvement.

Initial pus culture taken from a nodule turned out to be sterile. Gram staining and modified acid fast staining were done from the pus, which revealed Gram positive filaments with branching hyphae [Figure 5].

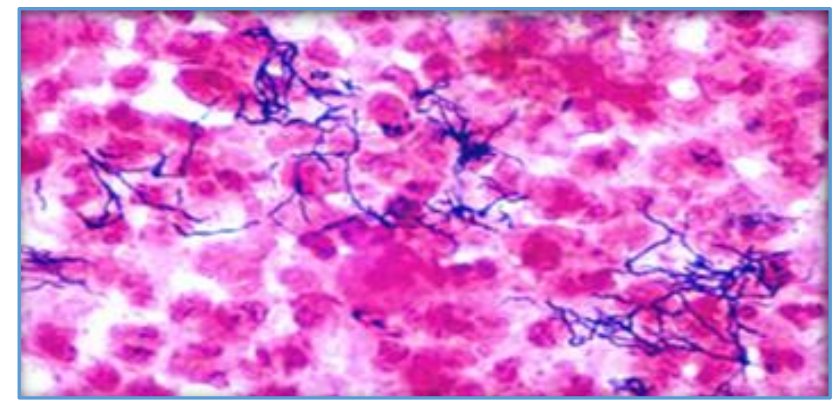

Figure 5. Gram Positive Delicate Filaments

A wedge biopsy done from a nodule revealed suppurating granulomas [Figure 6].

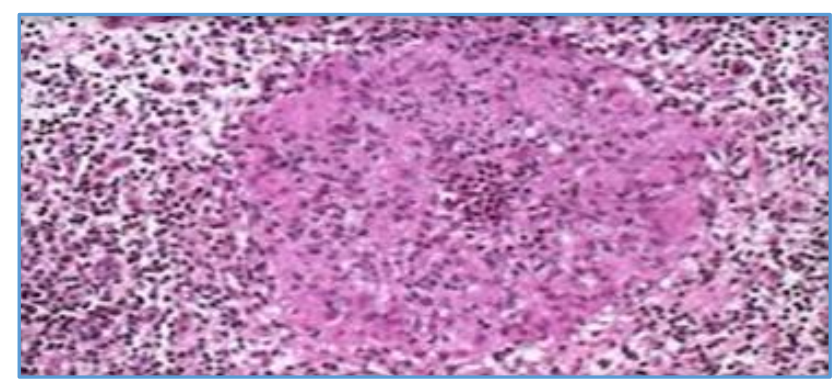

Figure 6. Histopathology shows Suppurative Granulomas

An abscess was surgically drained and aspirates were sent for repeat pus culture. Culture was done on a Sabouraud's agar medium (SDA) enriched with blood agar which yielded multiple, compact creamy white colonies of Nocardia asteroides [Figure 7].

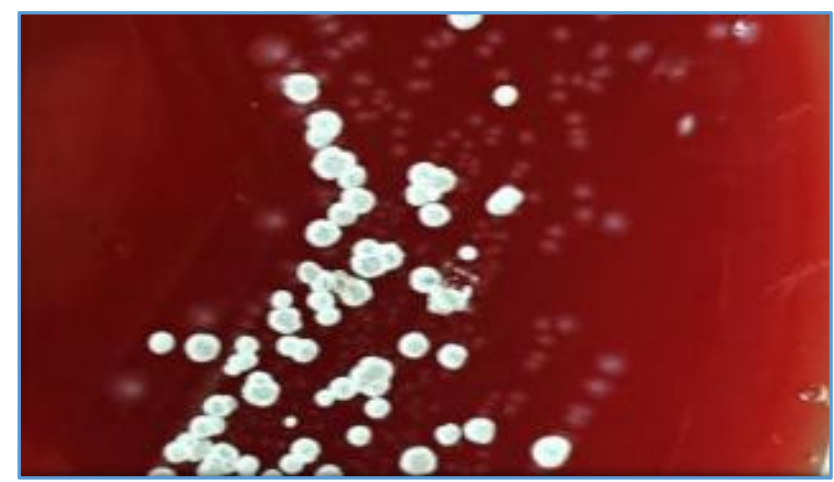

Figure 7. All these Features were Characteristic of Nocardia Asteroides and hence a Diagnosis of Primary Cutaneous Nocardiosis was Made
The two larger nodules were surgically excised and the patient was started on Cotrimoxazole. Lesions began to heal in two weeks' time. Two months after starting the treatment, there was complete resolution of all the lesions. But treatment was continued for six months with regular monthly followup. Relapse was noticed one month after stopping the treatment and hence the treatment was continued for another six months [Figure 8].

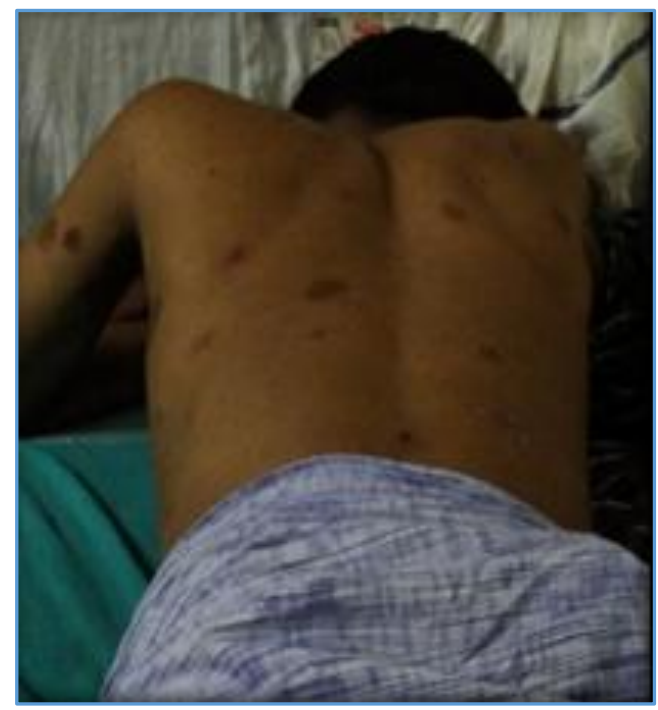

Figure 8. Healed Cutaneous Lesions with PostInflammatory Pigmentation

\section{FINAL DIAGNOSIS}

Fungal infections produce diverse clinical features that pose difficulty in diagnosis and management. Nocardiosis is an acute or chronic, suppurative, granulomatous disease caused by members of the genus Nocardia. Nocardia species are aerobic actinomycetes living free in nature. Nocardia asteroides is the predominant human pathogen. Other species include N. brasiliensis, N. otitidiscaviarum and N. farcinica. ${ }^{1}$

Most infections follow inoculation of the fungus from soil or wood into the abraded skin to cause localised actinomycotic mycetoma. The infection can present as nodules, pustules, abscesses and rarely lymphocutaneous syndrome. In few cases, dissemination have been reported. Their inhalation would result in primary pulmonary disease and they can eventually metastasize to any part of the body. ${ }^{2}$

Nocardiosis has been reported from all over the world. However, in India, the number of reports of Nocardia infection is limited. ${ }^{3}$

\section{We Report this Case due to its Rarity and following Unusualities:}

Here, our patient is a young healthy male with a sedentary lifestyle without any comorbidities or immunosuppression. The initial lesions started as tender nodules on the face and neck without any systemic dissemination. Due to these unusualities, the condition was wrongly diagnosed at two different hospitals. The delay in diagnosis led to wrong treatment and suppuration of the nodules. Hence, physicians should always consider subcutaneous fungal infections as a differential diagnosis of nodules and abscess, especially if they fail to respond to the usual treatment modalities. 


\section{REFERENCES}

[1] Ajantha GS, Kulkarni RD. Cladophialophora bantiana, the neurotropic fungus-a mini review. J Clin Diagn Res 2011;5:1301-6.

[2] Shivaswamy KN, Pradhan P, Laxmisha C, et al. Disseminated pheohyphomycosis. Int J Dermatol 2007;46(3):278-81.
[3] Sharma NL, Mahajan V, Sharma RC, et al. Subcutaneous pheohyphomycosis in India--a case report and review. Int J Dermatol 2002;41(1):16-20. 